\title{
Instrument concepts for the observation of prominences with future ground-based telescopes
}

\author{
A. López Ariste \\ THEMIS - CNRS UPS 853 \\ C/ Vía Láctea s/n 38205 - La Laguna, Spain \\ email: arturo@themis.iac.es
}

\begin{abstract}
The observation of prominences with ground-based telescopes suffers from poor image quality due to atmospheric turbulence when compared with space-borne instruments which, for solar observations, are of similar apertures. To make ground-based instruments competitive, they should rely on spectropolarimetry and the measurement of prominence magnetic fields, a task which no foreseable space instrument will perform. But spectropolarimetry alone does not suffice, and we argue that future instrumentation should combine it with imaging in a large field of view and good temporal resolution. We place numbers on those requirements and give examples of instrumental accomplishments already at work today that forecast a new generation of instruments for the observation of prominences from ground-based telescopes.
\end{abstract}

Keywords. Instrumentation; Polarimetry; Imaging

\section{Introduction}

The difficulties of observing the Sun force solar telescopes to small apertures, limited by the ability of materials and optical design to control heat. This constraint has a curious downside for ground-based solar telescopes, and it is that space solar telescopes have similar sizes too. Ground-based solar telescopes do not have the advantage of size over their space counterparts that night telescopes may enjoy.

What is left for ground-based solar telescopes to do when the advantage of size disappears?

This crucial question underlines the almost day-to-day prospective of future instrumentation for ground-based solar telescopes. It is clear that space telescopes, observing from beyond the disturbing effects of the atmospheric turbulence, enjoy an almost-perfect image of the Sun, constant in quality over the observing time. Until recently it could be asserted that the best spots on Earth could still take advantage of the larger mirror sizes and of performant adaptive optics systems to, from time to time, produce the highestresolution images possible of the solar atmosphere. The best images and movies from the Swedish Solar Telescope in La Palma with its $1 \mathrm{~m}$ aperture apparently outperformed the best images coming from the largest space solar telescope, Hinode, with its $0.5 \mathrm{~m}$ aperture. Recent results by van Noort(2012) and Ruiz Cobo and Asensio Ramos (2013) deconvolving images from the SOT instrument aboard Hinode cast a doubt over this general idea and future space solar telescopes (such as the projected Solar-C with its 1.5 aperture) will put further pressure on the performance of adaptive optics for new ground-based solar telescopes like ATST (Advanced Technology Solar Telescope) and EST (European Solar Telescope), a performance already pushed to the limit by the large aperture $(4 \mathrm{~m})$ of these future telescopes. For the observation of solar prominences, the advantage in image 
quality falls almost completely on the side of space telescopes. Adaptive optics systems require some light and a contrasted image to work. Those requirements are fulfilled over the solar disk where the observation of filaments with ground-based solar telescopes can still enjoy some good image quality under exceptional conditions (Lin et al., 2003 and Lin et al., 2005). Off-limb prominences on the other hand do not have the conditions for image correction, and in spite of some impressive examples when contrasted objects could be seen in the adjacent solar disk under conditions of large isoplanatic angles (Martínez González et al., 2014), high resolution images of prominences are the the realm of space solar telescopes (Berger et al., 2008, Chae et al., 2008).

The question returns therefore to what is left for ground-based telescopes to do when observing prominences, in a time when the Hinode/SOT instrument has provided spectacular observations of prominences that have renewed the debate as to how prominences are formed and about the relation of the observed dynamics with its magnetic structure.

\section{Spectra, polarization and imaging}

A first answer is spectropolarimetry. At this date the measurement of magnetic fields in prominences and filaments through spectropolarimetry of the He lines is a exclusivity of just 3 telescopes around the world, all ground-based: THEMIS, the DST and the VTT through its TIP instrument. Spectropolarimetry is still a complex technique. It requires a combination of powerful spectrographs and reliable and highly sensitive polarimeters. However, its complexity arises mostly from the difficulty of its interpretation. The constraint to only two He lines (the $\mathrm{He}_{3}$ line and the He line at $1083 \mathrm{~nm}$ ) is due to the fact that only those allow a simple enough treatment of the polarized line formation in the presence of quantum coherences, a requirement for the Hanle effect on which the diagnostic is based (López Ariste \& Casini, 2002). These difficulties make the technique not a subject of choice for space instruments which are characterized by their request for reliability and the absence of doubts on the interpretation of the raw measurement. Because of this no space instrument has ever carried a spectropolarimeter capable of these measurements, however important they are. Ground based solar telescopes observing prominences can profit from the ability to do spectropolarimetry in these He lines in order to be competitive when compared with space solar telescopes. A recent and dramatic example of this is the result published by Schmieder et al. (2013). In their work the authors use the Hinode SOT imaging instrument to provide them with a time series of high quality images of a prominence in which a wave-like perturbation appears to propagate upwards. This observation would not be surprising or new were it not for the fact that, simultaneously, THEMIS measured the magnetic field over the prominence revealing a perfectly horizontal field $7 \mathrm{G}$ strong. The image quality of the THEMIS data leaves much to be desired. But this is not a problem since the interest on THEMIS data is NOT on its image quality, which is provided by the space-borne Hinode and almost impossible to improve upon with existing ground-based instruments. The strength of THEMIS data rests upon its spectropolarimetric capabilities added to the ability to infer from them the magnetic field vector all over the prominence. This is where the ground-based solar telescope, THEMIS in that case, outperformed the space instrument and justified its use.

Spectropolarimetry is therefore an essential ingredient on future instruments for the observation of prominences with ground-based telescopes.

Many advances in prominence observations have resulted from the ability to measure and interpret the dynamics of the plasma confined in the prominence (as examples, Berger et al., 2008, and Chae et al., 2008). The ability to do imaging in adequate spectral ranges quick enough to allow for the follow up of the movements and perturbations 

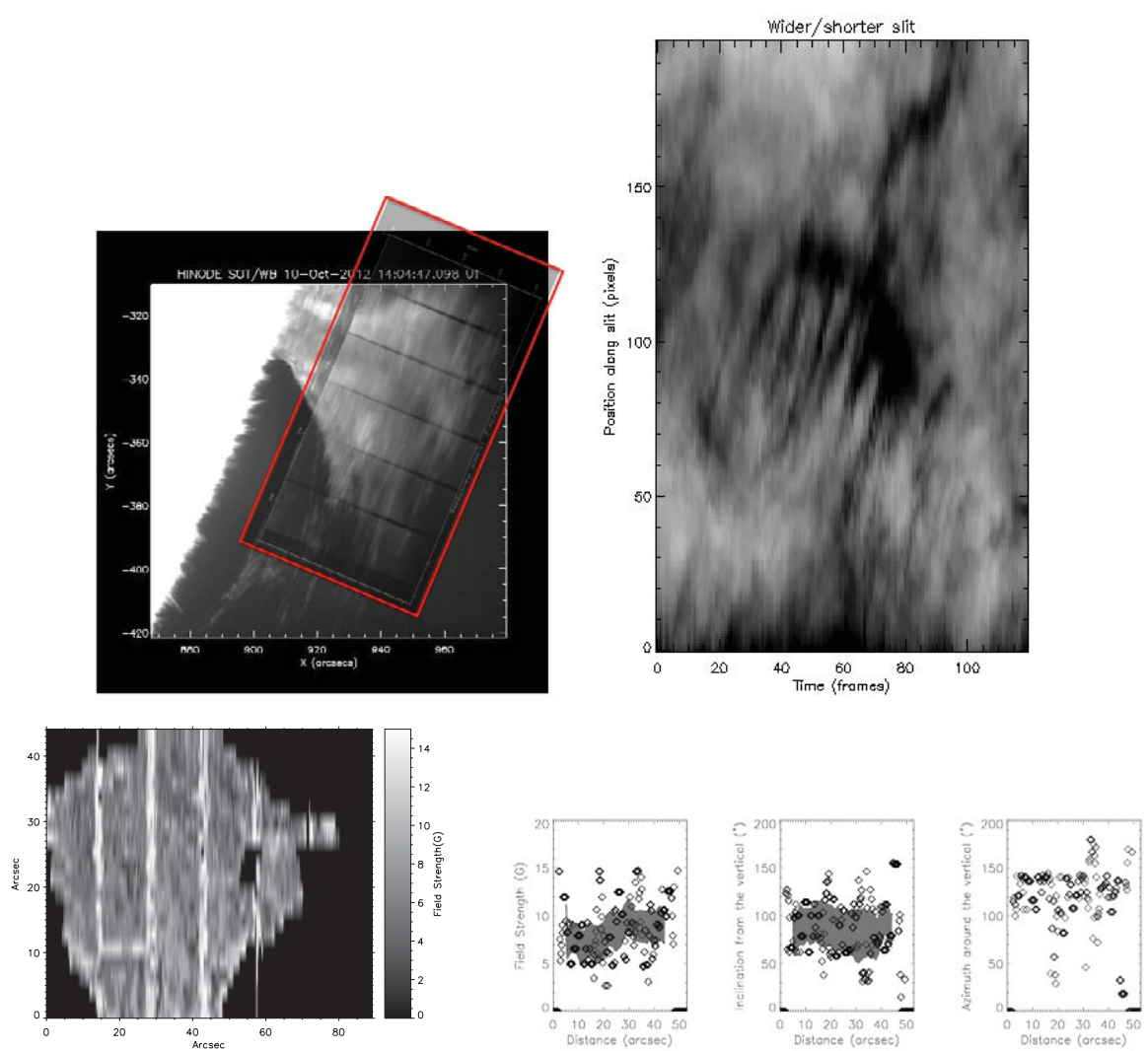

Figure 1. Collage of figures from Schmieder et al. (2013) illustrating space vs ground-based observations of prominences. The top images come from Hinode and show the prominence as seen in $\mathrm{H} \alpha$ and a time-distance diagram of a cut through the foot of the prominence. The red rectangle on the left image shows the region scanned with THEMIS. The bottom images show the magnetic field measurements made with the He $D_{3}$ line at THEMIS: the field strength over the prominence at left and the values of strength, inclination and azimuth of the magnetic field vector along the same cut through the prominence foot on which the time-distance diagram was made.

of the prominence plasma has shown the presence of counterstreaming threads, prominence oscillations of different kinds, or the presence of bubbles and plumes deforming the magnetic structure of the prominence. A very recent instrument focusing in this kind of observations from the ground is ROSA (Jess et al., 2010). Rather than giving the details of this instrument and its performances, it is interesting for the purpose of our present exercise to just look into the acronym that makes the name of this instrument: ROSA stands for Rapid Oscillations in the Solar Atmosphere. High quality imaging, we said, is the realm of space instruments. But as ROSA, and those recent observational results point out any future instrument must be capable of high cadence high quality imaging too. With this in mind, we have collected the three requirements for new instruments observing prominences: spectropolarimetry (in particular of the He lines) combined with imaging at sufficiently high cadence and quality as to follow the evolution, dynamics and oscillations of the prominence plasma. We should now give some numbers to make some sense of those three requirements.

Our first requirement for new instruments observing prominences was spectropolarimetry. The He lines $\mathrm{D}_{3}$ (at $587 \mathrm{~nm}$ ) and at $1083 \mathrm{~nm}$ are today the ones whose formation and 


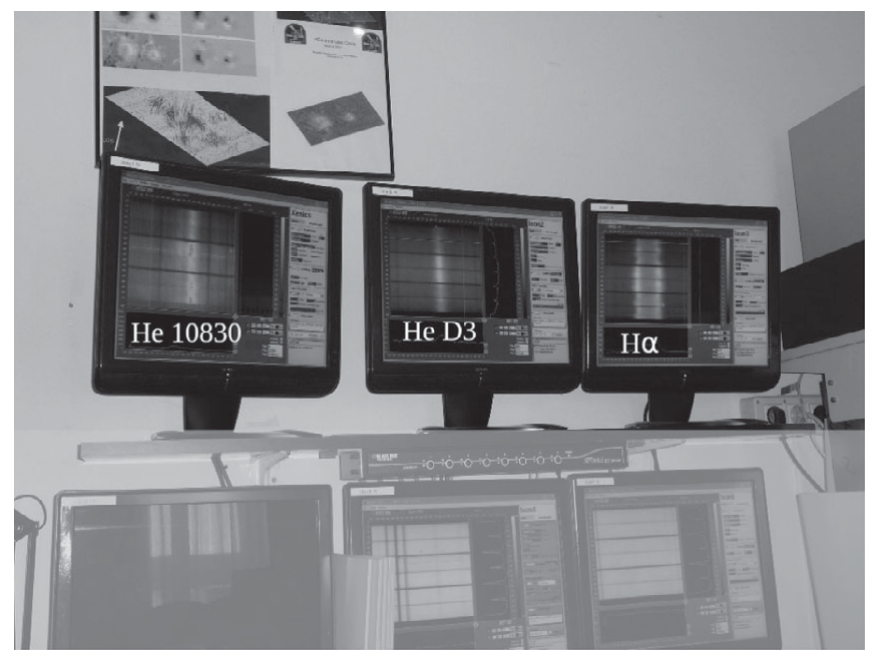

Figure 2. Observing prominences simultaneously in the 2 He lines at 587 and $1083 \mathrm{~nm}$ plus $\mathrm{H} \alpha$ with THEMIS.

polarization in the conditions of prominences and in the presence of magnetic fields is better understood. Our prospective instrument should therefore be able to observe either one of those spectral ranges. A much better constraint on the measured magnetic fields and other physical conditions is obtained if both of these lines are simultaneously observed and, furthermore, if a line like $\mathrm{H} \alpha$ is put in the set as a means to obtain a good feeling on the prominence morphology thanks to its large brightness. Observations with the three lines cited have already been performed, e.g. at THEMIS (see Fig. 2). Concerning spectral resolution, all recent measurements of magnetic fields in prominences have been done at spectral resolutions $\frac{\lambda}{\Delta \lambda}$ of around 200,000 though probably one can relax this constraint and retrieve reliable magnetic fields from polarimetry of those lines with values as low as 100,000. The linear polarization expected in these lines can be as high as $10 \%$, but it has been demonstrated time and again the importance of a reliable diagnostic for measuring the weak circular polarization in these lines, either due to the Zeeman effect or, as in the $\mathrm{D}_{3}$ line, the subtle transfer of atomic alignment into orientation. Those weak polarization signals peak at most at $0.1 \%$ of the intensity. To observe them, a requirement of a signal-to-noise ratio approaching 10,000 must be imposed on the instrument.

Our second requirement was imaging. The spatial resolution will be limited by the performance of the adaptive optics systems and, in the case of off-limb observations, the ability to use them off-limb. It is clear that space instruments will almost always outperform any ground-based instrument combining imaging with spectropolarimetry. Furthermore, signal-to-noise requirements, as given above, may require spatial resolution to be dropped in exchange for the accumulation of photons in every single detector pixel. Because of those reasons it is advisable not to set stringent requirements on spatial resolution. Another story is the field of view. The typical size of prominences must be observed to correctly interpret the morphology and, eventually, the temporal evolution. A minimum requirement of $1 \times 1 \operatorname{arcmin}^{2}$ with a goal to $2 \times 2 \operatorname{arcmin}^{2}$ appears as a reasonable choice. 
Finally, we are left with the third requirement of temporal resolution. Here our limitations come from both the required time to perform full polarimetry with the requested signal-to-noise ratios and also the ability to collect and handle the data at high rates. On the other hand we are required to observe, not the evolution of the prominence topology which more often than not happens in times of hours, but faster events as waves and oscillations which occur in periods of minutes (Ballester et al., 2012, Arregui et al., 2012). A prospective instrument should aim at temporal cadences of less than one minute therefore.

The two previous paragraphs almost look like a specification list for a new instrument. And in a basic sense they are so. The level of details given is chosen on purpose. Requiring spectropolarimetry plus imaging plus temporal resolution would seem at first sight as an impossible dream. Putting numbers into those broad requirements helps us identify priorities and where instrumental trade-offs can help solve the problem of a do-it-all instrument. It should be stressed once again that the purpose of this paper is not to consider what new observations of solar prominences can be done in the absolute, but what observations can be done from ground-based telescopes that are competitive respect to its space counterparts. The requirement for spectropolarimetry plus imaging plus temporal resolution is justified by these comparisons. Only by satisfying those requirements can we expect instruments in ground-based solar telescopes to offer new observations on prominences.

But can those requirements be satisfied at all by a single instrument? We think that, with the figures given, the answer is almost afirmative. The ROSA instrument is an obvious answer to almost all of those requirements assuming that another choice of observed spectral regions can be implemented to better suit prominence observations. Here we should end with another attempt in the same direction:TUNIS, being commissioned at this time on THEMIS. TUNIS stands for Tunable Universal Narrowband Imaging Spectrograph. It is an imaging instrument with a field of view of $2 \times 2 \operatorname{arcmin}^{2}$ and a pixel sampling of 0.2 arcsec. It is based upon the concept of subtractive double pass, on which a spectrograph is used to select the spectral range and resolution. This concept has been used before very successfully in the Multi-Subtractive Double Pass (MSDP) suite of instruments (Mein, 2002). The usual long and narrow slit at the entrance of classic spectrographs disappears, allowing the said field of view into the spectrograph. In the first pass each point of that field of view is dispersed in wavelength. A spectral slit selects the appropriate spectral bandpass for each point. Because of the different angle of entrance into the spectrograph there will be a linear variation across the image of what wavelength is selected, but the bandwidth will be fixed by the spectral slit. In the present TUNIS instrument this bandwidth is set at $20 \mathrm{~m} \AA$ resulting in a maximum of 300,000 spectral resolution. The second pass through the spectrograph in subtractive mode ensures that the residual dispersion of $20 \mathrm{~m} \AA$ does not degrade image quality. Spectral coverage at each point has to be then performed by some wavelength scanning. Rather than just scan the spectrum, though, TUNIS relies on wavelength multiplexing with a Hadamard mask. With this method, each point of the image sees in one exposure a linear combination of the intensities at 63 different wavelengths $20 \mathrm{~m} \AA$ wide. By making different linear combinations of the kind in subsequent exposures one can solve the linear system and retrieve the intensity of each individual wavelength during data reduction. In the present TUNIS, this can be done in about 2 seconds without polarimetry. More details of this instrument have been given by López Ariste et al. (2010) and López Ariste et al. (2011). The work in progress with instruments like ROSA and TUNIS demonstrates in our view that the requirements given above can be satisfied, aside from small concessions concerning, for example, spectral scanning. 


\section{Conclusion}

Summarizing, the observation of solar prominences with ground-based solar telescopes should rely mostly on spectropolarimetry and magnetic field measurements, the realm on which space instruments cannot compete. Added to that requirement, they should of course aim at also adding imaging and temporal resolution. Since in neither of these two aspects ground-based instruments can hardly outperform space instruments, they cannot be the priority of our ground-based instruments but should be seen as the perfect addition to spectropolarimetry to give ground-based instruments a place in the upcoming evolutions of our understanding of solar prominences. If the addition of spectropolarimetry plus imaging plus temporal resolution may seem an impossible task to achieve, when actually giving numbers on the actual requirements we see that the task can be actually accomplished with few concessions. Actually we think that two recent instruments as ROSA and TUNIS on the DST and THEMIS telescopes respectively are actually almost satisfying those requirements.

\section{References}

Arregui, I., Oliver, R., \& Ballester, J. L., 2012. Prominence Oscillations. Living Reviews in Solar Physics 9, 2.

Ballester, J. L., Arregui, I., Oliver, R., Terradas, J., Soler, R., Lin, Y., Engvold, O., Langagen, O., \& Rouppe van der Voort, L. H. M., 2012. Prominence seismology using ground- and space-based observations, pp. 169-174.

Berger, T. E., Shine, R. A., Slater, G. L., Tarbell, T. D., Title, A. M., Okamoto, T. J., Ichimoto, K., Katsukawa, Y., Suematsu, Y., Tsuneta, S., Lites, B. W., \& Shimizu, T., 2008. Hinode SOT Observations of Solar Quiescent Prominence Dynamics. The Astrophysical Journal Letters 676, L89-L92.

Chae, J., Ahn, K., Lim, E. K., Choe, G. S., \& Sakurai, T., 2008. Persistent Horizontal Flows and Magnetic Support of Vertical Threads in a Quiescent Prominence. The Astrophysical Journal Letters 689, L73-L76.

Jess, D. B., Mathioudakis, M., Christian, D. J., Keenan, F. P., Ryans, R. S. I., \& Crockett, P. J., 2010. ROSA: A High-cadence, Synchronized Multi-camera Solar Imaging System. Solar Physics 261, 363-373.

Lin, Y., Engvold, O., Rouppe van der Voort, L., Wiik, J. E., \& Berger, T. E., 2005. Thin Threads of Solar Filaments. Solar Physics 226, 239-254.

Lin, Y., Engvold, O. R., \& Wiik, J. E., 2003. Counterstreaming in a Large Polar Crown Filament. Solar Physics 216, 109-120.

López Ariste, A., \& Casini, R., 2002. Magnetic Fields in Prominences: Inversion Techniques for Spectropolarimetric Data of the He I D3 Line. Astrophysical Journal 575, 529-541.

López Ariste, A., Le Men, C., \& Gelly, B., 2011. Double-pass spectroimaging with spectral multiplexing: TUNIS. Contributions of the Astronomical Observatory Skalnate Pleso 41, 99-105.

López Ariste, A., Le Men, C., Gelly, B., \& Asensio Ramos, A., 2010. Double-pass spectroimaging: TUNIS. Astronomische Nachrichten 331, 658.

Martínez González, M., Manso Sainz, R., Asensio Ramos, A., Beck, C., \& De La Cruz Rodríguez, J., 2014. Observation of the magnetic field in solar tornadoes.

Mein, P., 2002. The MSDP of THEMIS: Capabilities, first results and prospects. Astronomy and Astrophysics 381, 271-278.

van Noort, M., 2012. Spatially coupled inversion of spectro-polarimetric image data. I. Method and first results. Astronomy and Astrophysics 548, 5 .

Ruiz Cobo, B., \& Asensio Ramos, A., 2013. Returning magnetic flux in sunspot penumbrae. Astronomy and Astrophysics 549, L4.

Schmieder, B., Kucera, T., Knizhnik, K., Luna, M., López Ariste, A., \& Toot, D., 2013. Propagating Waves Transverse to the Magnetic Field in a Solar Prominence. Astrophysical Journal 777, 108. 